\title{
Optimization of Magnetooptic Device by Low Switching Field Domains
}

\author{
Jin-Wei Tioh, Sasha Oster, Mani Mina, and Robert J. Weber \\ Department of Electrical and Computer Engineering, Iowa State University, Ames, IA 50011, USA \\ Correspondence should be addressed to Jin-Wei Tioh, jinwei@iastate.edu
}

Received 19 December 2011; Accepted 9 January 2012

Academic Editors: J. M. Girkin and I. S. Moreno

Copyright () 2012 Jin-Wei Tioh et al. This is an open access article distributed under the Creative Commons Attribution License, which permits unrestricted use, distribution, and reproduction in any medium, provided the original work is properly cited.

This paper expounds on the optimization of magnetooptic devices using preferential domains that switch at low field strengths. In particular, an all-optical switch for transparent networks based on the Mach-Zehnder interferometer configuration is examined in detail. The switch utilizes bismuth-substituted iron garnets with a specific composition of $\left(\mathrm{Bi}_{1.1} \mathrm{~Tb}_{1.9}\right)\left(\mathrm{Fe}_{4.25} \mathrm{Ga}_{0.75}\right) \mathrm{O}_{12}$ as Faraday rotators. It is proposed that switch figures of merit can be improved by preferentially choosing domains which align with applied fields at field strengths much lower than required by the bulk material. Measurement of magnetic domain orientation in the material and Faraday rotation within domains is reported. The domain behavior in low magnetic fields is also investigated to achieve a switch with lower switching times and higher extinction ratios.

\section{Introduction}

Faraday experimentally observed a rotation of the plane of polarization when light was transmitted through glass in a direction parallel to that of an applied magnetic field in 1845 [1]. This constituted the first demonstration magnetooptics followed by the observation of a broadening of the spectral lines emitted by a sodium flame when it was placed between the poles of an electromagnet by Zeeman in 1896 [2]. Since these pioneering discoveries, magnetooptics has become a captivating field of research, finding a great many scientific and practical applications [3-6].

In optical networking, magnetooptic (MO) devices are a potential enabler of better scaling, transparent networks that are bit-rate, protocol, and format insensitive. Transparency is critical given the networking paradigm shift from connection-oriented communication to high bandwidth IP-centric packet switched data traffic driven by the influx of high bandwidth applications [7] (Figure 1). This is made more urgent by the large and growing optical-electronic bandwidth mismatch [8] and the fact that devices are fast approaching the quantum limit [9].

Among the contending implementation technologies for all-optical switches, MO devices promise to offer lower insertion losses and faster switching times with the recent advances in suitable candidate materials [10-12].

In this paper we examine how MO switching technologies can be improved through material investigation of the Faraday rotator. The MO properties of bismuth-substituted iron garnets (BIGs) are investigated at the domain level. In particular, we consider how the domain properties of the material can be used to increase switching speed and extinction ratio. There are a number of other existing applications that rely on the domain wall motion in MO materials for their operation $[13,14]$. It is proposed that domains which show favorable characteristics can be preferentially used in order to achieve switching or operation of other applications at much lower field strengths than previously reported.

\section{Switch Design}

The switch to be optimized is based on a Mach-Zehnder interferometer configuration as illustrated in Figure 2. An MO Faraday rotator (MOFR) is inserted in each interferometer leg to equalize their amplitudes to a first order as well as to affect a given amount of phase mismatch when the MOFRs are magnetically biased. 


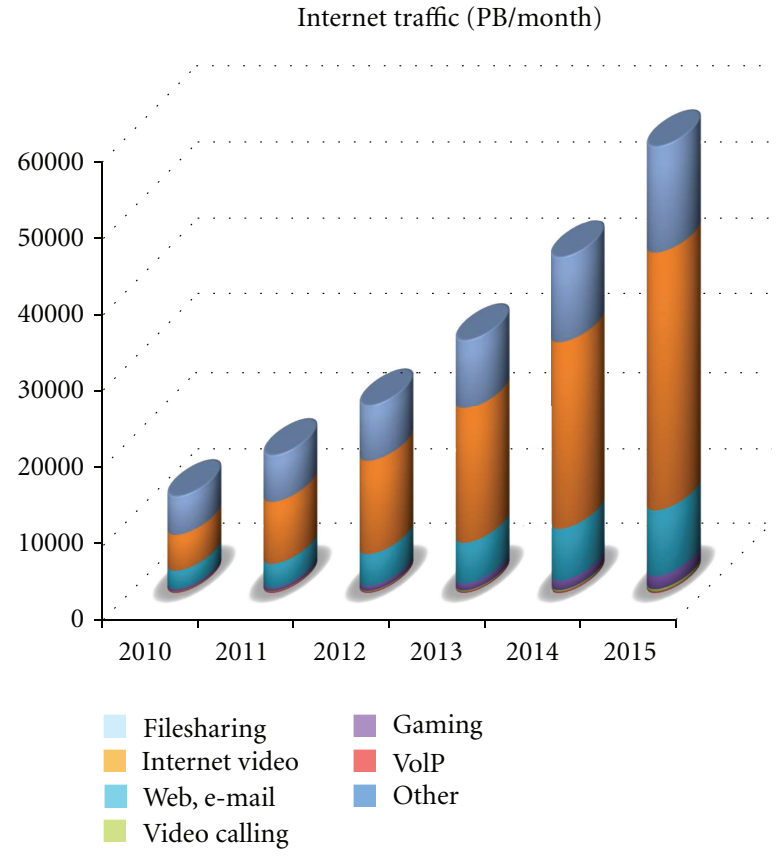

FIGURE 1: Forecasted growth of global IP traffic.

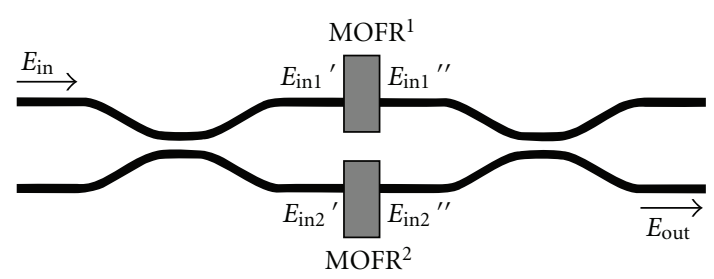

Figure 2: Structure of MO switch based on the Mach-Zehnder interferometer configuration.

At the switch input, a linearly polarized beam $\left(E_{\mathrm{in}}\right)$ is split with a 50/50 coupler. This produces two beams of equal amplitude with a $90^{\circ}$ phase difference $\left(E_{\mathrm{in} 1^{\prime}}\right.$ and $\left.E_{\text {in2 }}{ }^{\prime}\right)$, both of which can be decomposed into left- and right- circularly polarized components for propagation through the MOFR. This can be expressed by using the conversion operator:

$$
C=\frac{1}{2}\left[\begin{array}{cc}
1 & j \\
1 & -j
\end{array}\right] \text {. }
$$

Each component represents a different propagation eigenmode within the MOFR, with different effective refractive indices and propagation velocities within the material. This is captured by the propagation operator:

$$
W=e^{-j \varphi}\left[\begin{array}{cc}
e^{j \theta_{F}(t)} & 0 \\
0 & e^{-j \theta_{F}(t)}
\end{array}\right]
$$

where $\theta_{F}$ is the Faraday rotation angle and the common phase $\varphi$ is defined as:

$$
\varphi=\operatorname{Re}\left(\frac{\pi t\left(n_{+}+n_{-}\right)}{\lambda}\right)
$$

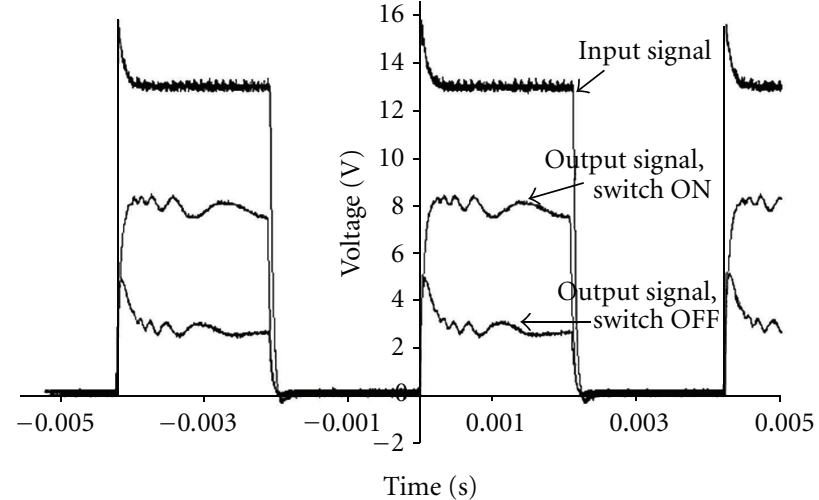

FIgURE 3: Measured extinction ratio for SMF-28.

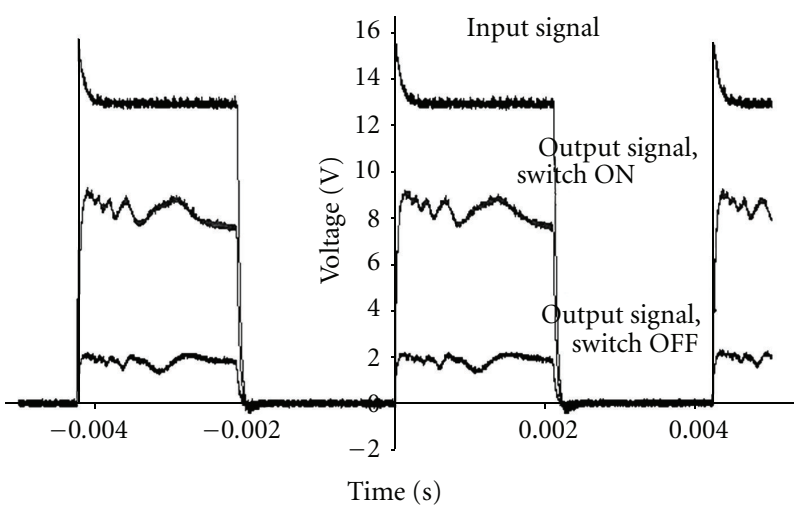

FIGURE 4: Measured extinction ratio for MMF.

The difference in propagation velocities is manifested as a rotation of the state of polarization (SOP) when the components recombine upon exiting the MOFR and can be expressed using standard Jones calculus [15] as:

$$
J_{\mathrm{FR}}=C^{-1} W C=\left[\begin{array}{cc}
\cos \left(\theta_{F}\right) & -\sin \left(\theta_{F}\right) \\
\sin \left(\theta_{F}\right) & \cos \left(\theta_{F}\right)
\end{array}\right] .
$$

When biased with a field $H_{\text {bias, }}$, the SOP of the beams from the first coupler $\left(E_{\mathrm{in} 1^{\prime}}\right.$ or $\left.E_{\mathrm{in} 2^{\prime}}\right)$ are rotated by $\theta_{F 1}$ and $\theta_{F 2}$, respectively,

$$
\left[\begin{array}{l}
E_{\text {in1-x }}^{\prime \prime} \\
E_{\text {in } 1-y}^{\prime \prime}
\end{array}\right]=\left[\begin{array}{cc}
\cos \left(\theta_{F 1}\right) & -\sin \left(\theta_{F 1}\right) \\
\sin \left(\theta_{F 1}\right) & \cos \left(\theta_{F 1}\right)
\end{array}\right]\left[\begin{array}{c}
E_{\text {in } 1-x}^{\prime} \\
E_{\text {in } 1-y}^{\prime}
\end{array}\right] .
$$

In the MOFR's linear region of operation, $\theta_{F}$ is described by $\theta_{\text {sat }}\left(H_{\text {bias }} / H_{\text {sat }}\right)$, where $\theta_{\text {sat }}$ is the rotation at the MO material saturation magnetic field. This relationship is valid when the beam passes through a sufficient number of domains and the applied field is below the saturation field.

Selecting an appropriate field value $\left(H_{\text {switch }}\right)$ to give a total phase mismatch of $180^{\circ}$ between the two interferometer arms $\left(\theta_{F 1}=90^{\circ}, \theta_{F 2}=-90^{\circ}\right)$ results in complete destructive interference at the switch output. Thus, the switch $\mathrm{ON}$ $\left(H_{\text {bias }}=0\right)$ and OFF $\left(H_{\text {bias }}=H_{\text {switch }}\right)$ states are achieved. Extinction ratios of $8.5 \mathrm{~dB}$ for SMF-28 fiber (Figure 3) and $12.5 \mathrm{~dB}$ MM fiber (Figure 4) have been empirically determined with $H_{\text {switch }}=16 \mathrm{kA} / \mathrm{m}$. 


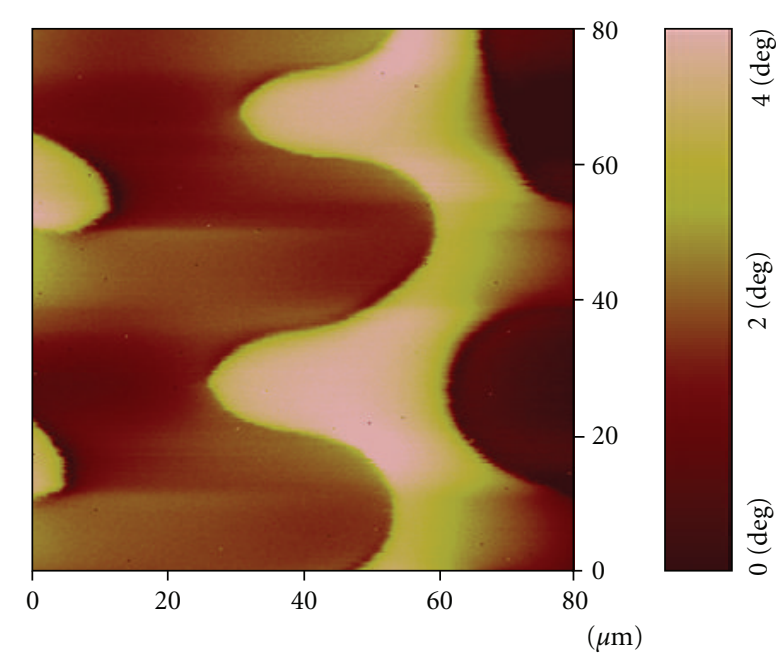

Figure 5: Magnetic force microscope image of the BIG domain structure.

The difference in extinction ratios can be attributed to the relationship between the beam and domain sizes. In the case of the switch realized with SMF-28 fiber, the action of the individual domains becomes the major contributing factor to the amount of Faraday rotation and thus the switch extinction ratio as well as the bias field magnitude required to achieve it.

The field generating coil is a multilayer solenoid with a quiescent current of $5 \mathrm{~A}$ and measured inductance of $1.2 \mathrm{mH}$. It is driven by four MC33886 H-bridges in parallel to provide a maximum bidirectional current drive of $20 \mathrm{~A}$ that can be pulse-width modulated up to $10 \mathrm{kHz}$.

The time constant associated with the coil overshadows the delay associated with the MOFR domain dynamics, since in principle, the velocity of the domain walls has been measured to be on the order of $10 \mathrm{~km} / \mathrm{s}$ [16]. More fundamentally, significant improvements in switching time can be achieved by reducing $H_{\text {switch }}$.

\section{Material Considerations}

BIGs provide a large amount of Faraday rotation per unit of material length and low absorption at optical frequencies, making them an attractive choice for use in optical devices. The BIG samples which are used as the MOFRs are $330 \mu \mathrm{m}$ thick with a refractive index of 2.344 at $1550 \mathrm{~nm}$ and $H_{\text {sat }}$ of $27.9 \mathrm{kA} / \mathrm{m}$. The bulk material rotates the SOP $45^{\circ}$ at $1550 \mathrm{~nm}$ with the saturation field applied.

The relationship $\theta_{F}=\theta_{\text {sat }}\left(H_{\text {bias }} / H_{\text {sat }}\right)$ is only valid when the diameter of the incident beam on the surface of the MOFR is sufficiently wide to sample a large number of domains. The BIG samples utilized have domain sizes of $20 \mu \mathrm{m}$ (Figure 5). The MM fibers used to realize the switch have a core diameter of $62.5 \mu \mathrm{m}$, sufficient to sample many domains and the MM switch therefore generally obeys the aforementioned relationship. However, when the beam size is on the scale of the domain size, local characteristics of the individual domains become the major contributing factor in the rotation of the SOP. It should be noted that these single-domain contributions are present even in the absence of an external field. This means there will be a rotation of the SOP of an incident beam even when an external field is not applied if the beam is predominantly confined to a single domain. The SMF-28 fibers used in the SM switch have a core diameter of $9 \mu \mathrm{m}$. Thus, the beam incident on the MOFR only samples a small number of domains or even a single domain and the SM switch experiences this effect. This phenomenon is subsequently utilized to locate the domains.

In this basic switch setup, the position of the $\mathrm{MO}$ is not chosen so that the beam exiting the fiber is aligned to preferential domains within the sample. Instead, the bulk Faraday rotation depends on for the rotation of the SOP. In an external field, the two main mechanisms for switching the magnetic domains are the rotation of individual magnetic moments and motion of domain walls [17]. In the presence of low fields, favorably aligned domain will begin to grow via domain wall motion, and at moderate field strengths, domains will rotate to their easy axes, according to the crystallographic structure, which most closely corresponds to the direction of the applied field [18]. Finally at high field strengths, the domains will align with the direction of the applied field.

This domain motion corresponds to the amount of rotation experienced by the beam. Faraday rotation increases as the magnetic moments of more domains grow and rotate toward the direction of the applied field. Some individual domains align with an applied magnetic field at much lower field strengths than the bulk material. Therefore, the speed of the switch at the material level is limited by how quickly the magnetic domains can grow and begin to align with an applied magnetic field.

Being a thick ferrimagnetic material, the domains of the MOFR used are aligned such that the magnetic moment of neighboring domains are antiparallel to each other [19] and perpendicular to the face of the MOFR (out of plane magnetization). The field is therefore applied perpendicular to the face of the material. By locating domains that switch with very low applied fields and confining the beam predominantly within one of these domains, switching can be accomplished at much lower fields. Generating a magnetic field of smaller magnitude will also have a smaller time constant associated with the inductance required to switch the field, so switching can be achieved more rapidly at the circuit level.

\section{Empirical Results}

In order to determine the size of the beam incident on the MOFR sample for a SMF-28 fiber, the lateral offset method was used with the setup as illustrated in Figure 6.

In this method, two SM fibers, a receiving and transmitting fiber, were aligned and the distance between them was minimized. The transmitting fiber was connected to a $1550 \mathrm{~nm}$ laser source. Both the transmitting and receiving fibers were coiled for a length to create a mode stripper that removed higher order modes in the fibers. With the 


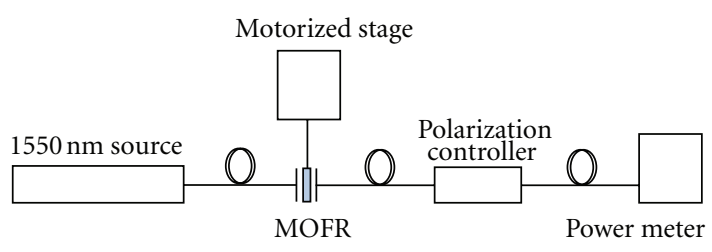

Figure 6: Experimental setup for locating preferential domains.

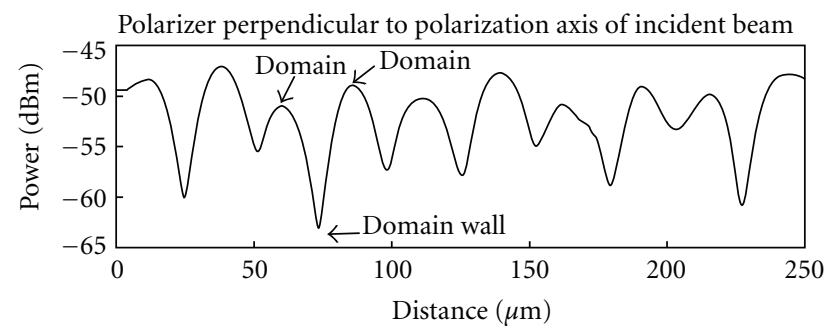

Figure 7: Domains located without regard to the orientation of the magnetic domains.

transmitting fiber held stationary, the receiving fiber was moved from left to right across the face of the transmitting fiber using a motorized stage. The receiving fiber was connected to an optical power meter and the power received was recorded as a function of position. Due to the incident and receiving pattern, for two near identical well-cleaved fibers, the resulting measured power is the convolution of two Gaussians. Due to reciprocity, these Gaussians can be represented as near identical and therefore the halfpower beam width (HPBW) of the incident beam can be determined.

With a separation of $1 \mathrm{~mm}$, the HPBW was measured to be approximately $16 \mu \mathrm{m}$. By using magnetic force microscopy, the width of the domains was determined to be approximately $20 \mu \mathrm{m}$ (see Figure 5 ). Therefore, in order to confine the beam, in terms of its HPBW to a domain, the separation between the face of the fiber and MOFR sample should be kept less than $1 \mathrm{~mm}$.

When the beam width incident on the MOFR is smaller than the domain dimensions, a linear source and polarizer can be used to locate domains in the material and the alignment of the magnetic moments of the domains can be determined. To do this, a MOFR was placed between the transmitting fiber, which was connected to an Agilent $81640 \mathrm{~A}$ $1550 \mathrm{~nm}$ linearly polarized laser source, and the receiving fiber, which was connected to an Agilent 8169 A polarization controller and in turn an Agilent 81635 optical power sensor. The MOFR was placed on a motorized stage and moved between the two fibers.

When the polarizer is set perpendicular to the axis of polarization of the incident beam the power received will be minimized through areas where the SOP undergoes no rotation (domain walls) and can be differentiated from areas where the beam undergoes rotation (domains). This is shown in Figure 7.

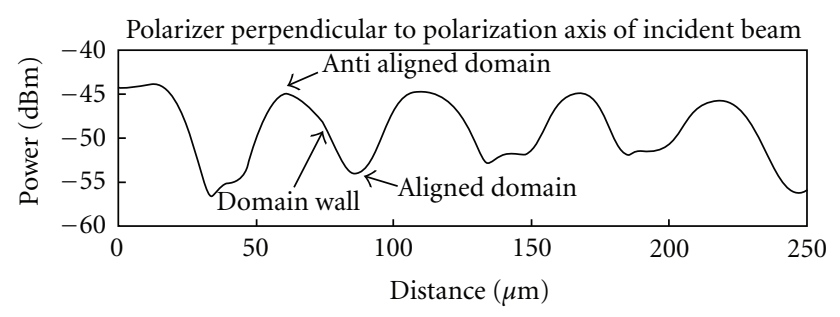

FIgURE 8: Orientation of the magnetic moments of the domains.

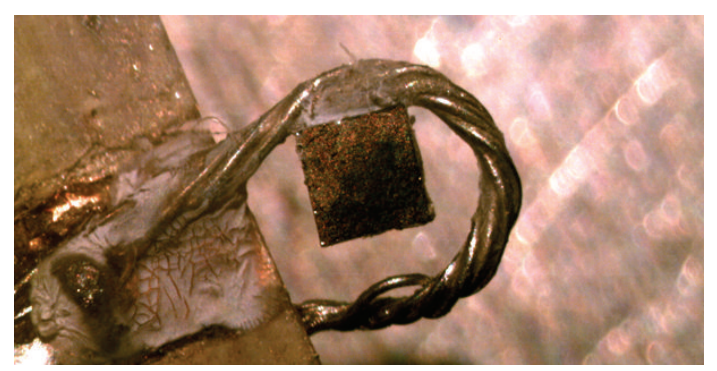

FIGURE 9: Field generating coil for characterizing the Faraday rotation of preferential domains.

The orientation of the domains can be determined by setting the polarizer perpendicular to the polarization axis of a beam after it has passed through an aligned domain as shown in Figure 8.

The Faraday rotation can also be measured within the domains. By aligning the fiber within a single domain and measuring the change of the polarization of the incident beam, the average magnitude of Faraday rotation in a domain in the absence of an external magnetic field was measured to be approximately $32^{\circ}$ with a standard deviation of $17^{\circ}$. In a small number of domains, Faraday rotation of $60^{\circ}$ or great was measured.

A coil was placed around the MOFR sample in order to generate a magnetic field through the material as shown in Figure 9. In low magnetic fields $(<2.39 \mathrm{kA} / \mathrm{m})$, there was no experimentally significant change in the Faraday rotation in most domains. However, in a very small number of domains, the magnetic moment of the domain switched and domain wall movement was measured. The magnetic moment of theses individual domain rotated with the applied field as low as $1.59 \mathrm{kA} / \mathrm{m}$, were under the saturation field of $27.85 \mathrm{kA} / \mathrm{m}$. This supports the theory that the magnetic moments of some domains will change at lower field strengths than the bulk material.

\section{Concluding Remarks}

A method of optimizing MO devices in general (and a Mach-Zehnder-interferometer-based MO switch in specific) has been described in detail. The domain behavior of the MOFR used in the switch was investigated and reported. Domains within a MOFR sample were differentiated and the orientation of their magnetic moments was identified. The Faraday rotation in the absence of a magnetic field was 
also measured and the behavior of the domains in low fields $(<2.39 \mathrm{kA} / \mathrm{m})$ was also examined. The alignment of a domain with an external magnetic field was achieved at low field strength for a small number of domains in the sample and enables faster switching speeds with lower power usage.

\section{References}

[1] M. Faraday, Experimental Researches in Electricity, vol. 3, London, UK, 1855.

[2] P. Zeeman, "The effect of magnetisation on the nature of light emitted by a substance," Philosophical Magazine, vol. 43, pp. 226-236, 1897.

[3] J. M. Kikkawa and D. D. Awschalom, "Resonant spin amplification in n-type GaAs," Physical Review Letters, vol. 80, no. 19, pp. 4313-4316, 1998.

[4] A. D. White, G. B. McHale, and D. A. Goerz, "Advances in optical fiber-based faraday rotation diagnostics," in Proceedings of the 17th IEEE International Pulsed Power Conference (PPC'09), pp. 1358-1363, July 2009.

[5] R. P. Panmand, G. Kumar, S. M. Mahajan et al., "Functionality of bismuth sulfide quantum dots/wires-glass nanocomposite as an optical current sensor with enhanced Verdet constant," Journal of Applied Physics, vol. 109, no. 3, Article ID 033101, pp. 1-7, 2011.

[6] Q. J. Gao, L. Li, and G. C. Niu, "Research on aircraft skin cracks recognition based on the crack-isolated method," in Proceedings of the International Conference on Biomedical Engineering and Computer Science (ICBECS'10), pp. 1-4, April 2010.

[7] Cisco Systems Inc., "Global IP traffic forecast and methodology 2010-2015," 2011.

[8] T. Sabapathi and S. Sundaravadivelu, "Analysis of bottlenecks in DWDM fiber optic communication system," Optik, vol. 122, no. 16, pp. 1453-1457, 2010.

[9] J. R. Powell, “The quantum limit to Moore's law," Proceedings of the IEEE, vol. 96, no. 8, Article ID 4567410, pp. 1247-1248, 2008.

[10] T. Okuda, T. Katayama, H. Kobayashi, N. Kobayashi, K. Satoh, and H. Yamamoto, "Magnetic properties of $\mathrm{Bi}_{3} \mathrm{Fe}_{5} \mathrm{O}_{12}$ garnet," Journal of Applied Physics, vol. 67, no. 9, pp. 4944-4946, 1990.

[11] S. Kang, S. Yin, V. Adyam, Q. Li, and Y. Zhu, " $\mathrm{Bi}_{3} \mathrm{Fe}_{4} \mathrm{Ga}_{1} \mathrm{O}_{12}$ garnet properties and its application to ultrafast switching in the visible spectrum," IEEE Transactions on Magnetics, vol. 43, no. 9, pp. 3656-3660, 2007.

[12] V. J. Fratello, S. J. Licht, and C. D. Brandle, "Compositional design of Faraday rotator materials, in multicomponent oxide films for electronics," in Proceedings of the Materials Research Society Symposium, vol. 574, pp. 225-236, 1999.

[13] S. J. Lee, Y. Melikhov, D. C. Jiles, C. M. Park, and H. Hauser, "Magneto-optic linear-displacement sensor with high spatial resolution and low noise," Journal of Applied Physics, vol. 99, no. 8, Article ID 08B301, pp. 1-3, 2006.

[14] S. Lee, Y. Melikhov, C. Park, H. Hauser, and D. C. Jiles, "Analysis of a remote magneto-optic linear displacement sensor using jones matrix approach," IEEE Transactions on Magnetics, vol. 42, no. 10, pp. 3273-3275, 2006.

[15] R. C. Jones, "A new calculus for the treatment of optical systems," Journal of Optical Society of America, vol. 31, no. 7, pp. 488-493, 1941.

[16] C. H. Tsang, R. L. White, and R. M. White, "Transit-time measurements of domain-wall mobilities in $\mathrm{YFeO}_{3}$," Journal of Applied Physics, vol. 49, no. 12, pp. 6052-6062, 1978.
[17] J. H. van Vleck, "Fundamental theory of ferro- and ferri- magnetism," Proceedings of the IRE, vol. 44, no. 10, pp. 1248-1258, 1956.

[18] D. C. Jiles, Introduction to Magnetism and Magnetic Materials, Boca Raton, Fla, USA, 2nd edition, 1998.

[19] M. Guillot, "Faraday rotation of bismuth substituted terbium iron garnets," IEEE Transactions on Magnetics, vol. 30, no. 6, pp. 4419-4421, 1994. 

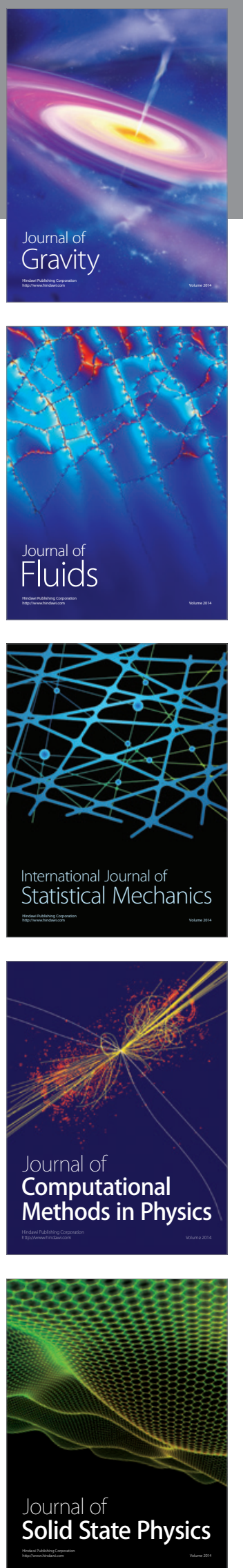
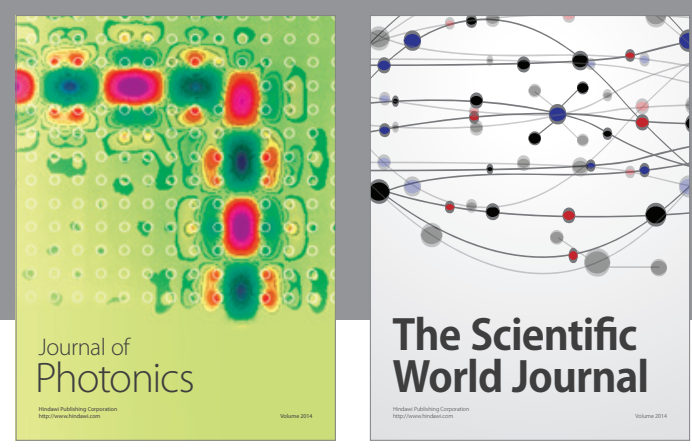

The Scientific World Journal

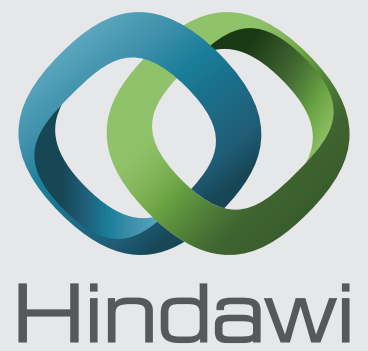

Submit your manuscripts at http://www.hindawi.com
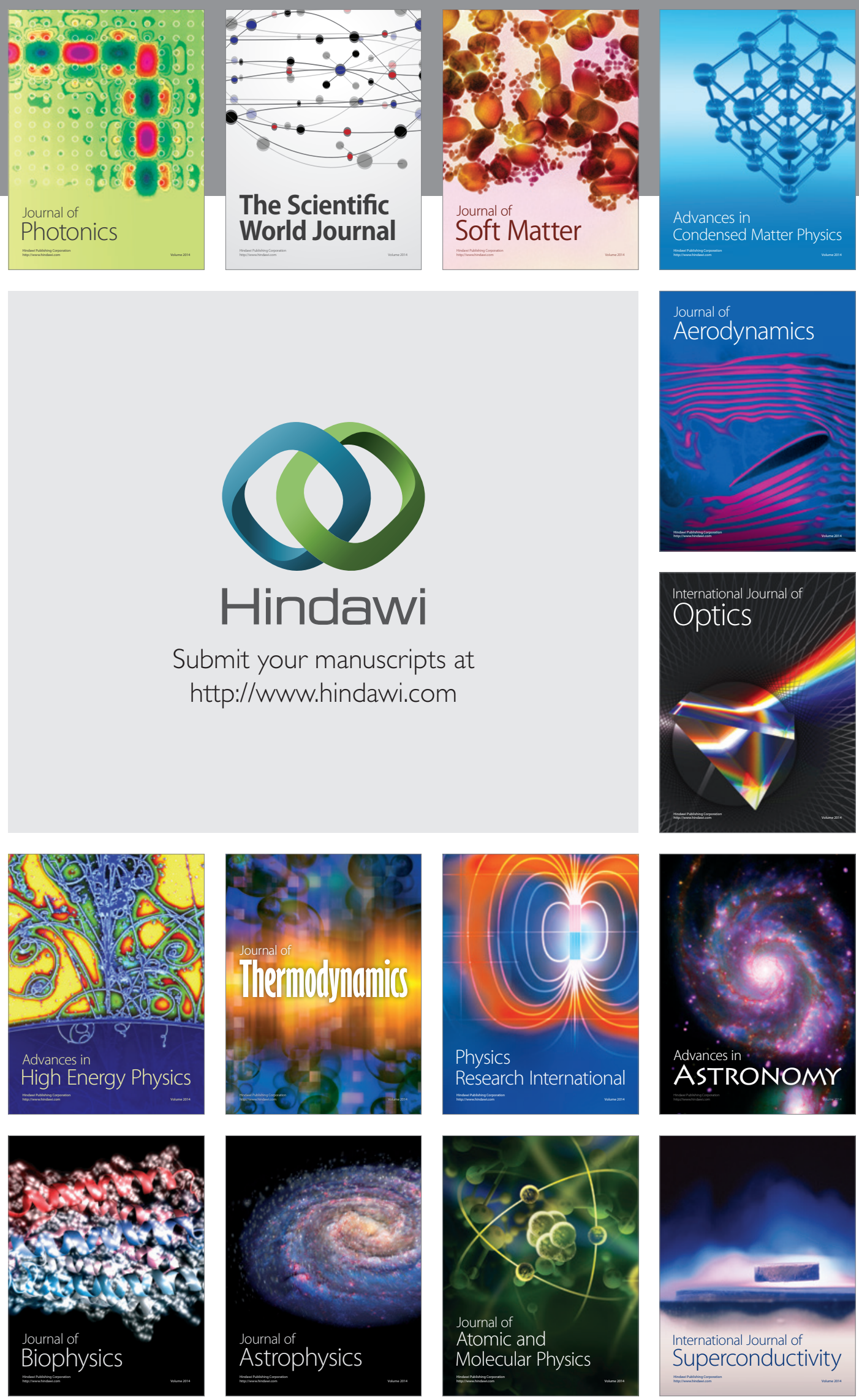
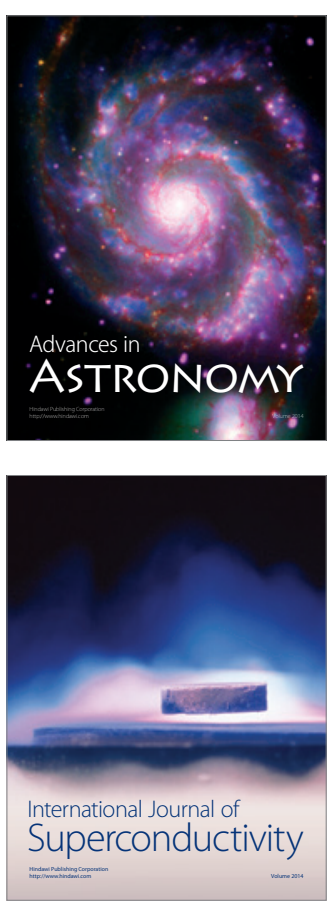